\title{
4D numerical analysis of scaffolds: a new approach
}

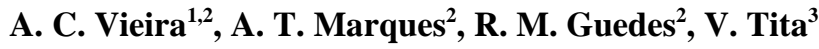

${ }^{1}$ UMEC, Institute of Mechanical Engineering and Industrial Management,

Rua Dr. Roberto Frias 378, 4200-465 Porto, Portugal

${ }^{2}$ DEMec, Mechanical Engineering Department, Faculty of Engineering of University of Porto (FEUP),

Rua Dr. Roberto Frias s/n, 4200-465 Porto, Portugal

${ }^{3}$ Aeronautical Engineering Department, Engineering School of São Carlos, University of São Paulo, Av. Trabalhador São-carlense, 400,13566-590, São Carlos, SP, Brazil

\begin{abstract}
A large range of biodegradable polymers are used to produce scaffolds for tissue engineering, which temporarily replace the biomechanical functions of a biologic tissue while it progressively regenerates its capacities. However, the mechanical behavior of biodegradable materials during its degradation, which is an important aspect of the scaffold design, is still an unexplored subject. For a biodegradable scaffold, performance will decrease along its degradation, ideally in accordance to the regeneration of the biologic tissue, avoiding the stress shielding effect or the premature rupture. In this chapter, a new numerical approach to predict the mechanical behavior of complex 3D scaffolds during degradation time (the $4^{\text {th }}$ dimension) is presented. The degradation of mechanical properties should ideally be compatible to the tissue regeneration. With this new approach, an iterative process of optimization is possible to achieve an ideal solution in terms of mechanical behavior and degradation time. The scaffold can therefore be prevalidated in terms of functional compatibility. An example of application of this approach is demonstrated at the end of this chapter.
\end{abstract}




\section{Introduction}

There are many biodegradable polymers commercially available to produce a wide variety of scaffolds, each of them with suitable properties, according to the tissue they are supporting during regeneration. Many examples can be found from generic tissue engineering scaffolds (Levenberg and Langer 2004), biodegradable ligaments (Vieira et al. 2009), biodegradable endovascular (Colombo and Karvouni 2000) and urethral stents (Tamela and Talja 2003). The design process must contemplate the biocompatibility issues related to toxicity and the functional aspect related to mechanical considerations. In terms of mechanical dimensioning, one must consider not only the static strength and stiffness of the device, but also the long-term mechanical behavior considering degradation. This degradation is defined as the time-dependent cumulative irreversible damage due to hydrolysis.

When loading conditions are simple and the desired time for mechanical support is known, a "trial and error" approach may be enough to design reasonable reliable scaffolds. In more complex situations, engineers and designers can use numerical approaches to define the material formulation and geometry that will satisfy the immediate needs of symptomatic relief, without the occurrence of any degradation, using conventional dimensioning. However, the lack of design tools to predict long term behavior has limited the success of biodegradable scaffolds. The considerations and the dimensioning methods developed until this moment may overcome this limitation, normally, providing a poor solution. Therefore, it is necessary to propose new approaches to improve the solution for this problem.

In this chapter, a new numerical approach, which can use hyper elastic constitutive models, such as the Neo-Hokean, the Mooney-Rivlin and the second reduced order will be discussed. In fact, the new approach consists on a constitutive model and a failure criterion, which are implemented in commercial finite element software packages like ABAQUS via User Material (UMAT) subroutine and Python language. Through a failure criterion, the degradation rate was used to define the strength of the material at a given degradation time, using a first order kinetic Equation. The material parameters of the constitutive model were calculated by inverse parameterization of the model compared against experimental data. It was found that only one material parameter varies linearly with the hydrolytic damage (which depends on the degradation time). Although this approach was evaluated based on experimental tensile tests of fibers, for a particular blend of polylactic acid (PLA) and polycaprolactone (PCL), the authors believe that this can be extended to other thermoplastic biodegradable materials with response similar to hyper elastic behavior. The new numerical approach was able to predict the loaddisplacement plot with reasonable accuracy until $50 \%$ of hydrolytic damage. It can be further extended to numerical 3D models and complex loading scenarios for different applications, to predict the long-term mechanical behavior. 


\section{Biodegradable Polymers}

Biodegradable polymers can be classified as either naturally derived polymers or synthetic polymers. A large range of mechanical properties and degradation rates are possible among these polymers. However, each of these may have some shortcomings, which restrict its use for a specific application, due to inappropriate stiffness or degradation rate. Blending, copolymerization or composite techniques are extremely promising strategies, which can be used to tune the original mechanical and degradation properties of the polymers (Aslan et al. 2000) according to the application requirements. The most popular and important class of biodegradable synthetic polymers are aliphatic polyesters, such as polylactic acid (PLLA and PDLA), polyglycolic acid (PGA), polycaprolactone (PCL), polyhydoxyalkanoates (PHA's) and polyethylene oxide (PEO) among others. They can be processed like other thermoplastic materials.

The poly-a-hydroxyesters, PLA, PGA and their copolymers are the most popular aliphatic polyesters that have been synthesized for more than 30 years. The lefthanded (L-lactide) and right-handed (D-lactide) are the two enantiomeric forms of PLA, with PDLA having a much higher degradation rate than PLLA, but similar initial mechanical properties. An exhaustive overview was done by Auras et al. (Auras et al. 2004). PLLA is a rather brittle polymer with a low degradation rate, and compounding with PCL is frequently employed to improve mechanical properties. PCL is also hydrophobic with a low degradation rate, much more ductile than PLA (Södergard and Stolt 2002). PGA, since it is a hydrophilic material presents a high degradation rate. It is stiffer than PLA. The combination of PGA with PLA is usually employed to tune degradation rate (Nair and Laurencin 2007). Polyhydoxyalkanoates (PHA's) is the largest class of aliphatic polyesters, comprising poly 3-hydroxybutyrate (PHB), copolymers of 3-hydroxybutyrate and 3hydroxyvalerate (PHBV), poly 4-hydroxybutyrate (P4HB), copolymers of 3hydroxybutyrate and 3-hydroxyhexanoate (PHBHHx) and poly 3hydroxyoctanoate (PHO) and its blends. The changing PHA compositions also allow favorable mechanical properties and degradation times within desirable time frames (Chen and Wu 2005). Natural polymers used in scaffolds include starch, collagen, silk, alginate, agarose, chitosan, fibrin, cellulosic, hyaluronic acid-based materials, among others. Some of these are bioactive materials, and their degradation products can modulate the inflammatory response. However, these are more prone to enzymatic degradation than the synthetic biodegradable polymers, consequently the degradation kinetics depends more on the host. The synthetic polymers have that advantage of more predictable behavior evolution. New biodegradable material solutions are continuously arising each day.

Presently 3D scaffolds can be printed with layers and parts of different biodegradable materials. These can then be coated with a biodegradable and bioactive material, to obtain a more intelligent surface in terms of cell mediated interaction. A complex geometry like these can be modeled in a commercial $3 \mathrm{D}$ drawing software and the mechanical behavior can be predicted by numerical simulation. 


\section{Biodegradation and Erosion}

All biodegradable polymers contain hydrolysable or oxydable bonds. This makes the material sensitive to moisture, heat, light and also mechanical stresses. These different types of polymer degradation mechanisms (photo, thermal, mechanical and chemical degradation) can be present alone or combined, working synergistically to the degradation. Usually, the most important degradation mechanism of biodegradable polymers is chemical degradation via hydrolysis or enzymecatalyzed hydrolysis (Göpferich 1996). The most important factor is its chemical structure and the occurrence of specific bonds along its chains. Like those in groups of esters, amides, etc., which might be susceptible to hydrolysis when exposed to water (Nikolic et al. 2003; Herzog et al. 2006).

Another important distinction must be made between erosion and degradation. Both are irreversible processes. However, while the degree of erosion is estimated from the mass loss, the degree of degradation can be estimated by measuring the evolution of molecular weight, by SEC (Size Exclusion Chromatography) or GPC (Gel Permeation Chromatography), or the tensile strength evolution (by universal tensile testing). Hence, the hydrolytic degradation process is included on the erosion process.

The erosion process can be described by phenomenological diffusion-reaction mechanisms presented in Figure 1. An aqueous media diffuses into the polymeric material while oligomeric products diffuse outwards to be then bio-assimilated by the host environment. Then, there is material erosion with correspondent mass loss. On the other hand, degradation refers to mechanical damage and depends on hydrolysis. Within the polymeric matrix, hydrolytic reactions take place, mediated by water and/or enzymes. While water diffuses rapidly well inside the material, enzymes are large molecules unable to do it, and so they degrade at surface. The degradation of polyesters by micro-organisms is initiated by extracellular hydrolases, which are secreted by the organisms to reduce the molar mass of the polymeric substrate and to make it bio-available and bio-assimilated. It was demonstrated by Tokiwa and Suzuki (Tokiwa and Suzuki 1977) that synthetic polyesters can be attacked by hydrolases (lipases). However, for most biodegradable materials, especially synthetic polymers, passive hydrolysis due to the presence of water molecules is the most important mode of degradation.

To fully model the erosion process, a complex mathematical model is needed to account for all the reaction steps and for the structural and morphologic details. The parameters in such a model require extensive experimentation. Numerical techniques have been used (Göpferich and Langer 1993; Wang et al. 2008; Yu et al. 2008; Han and Pan 2009; Bikiaris et al. 2007; Metzmacher et al. 2007) to solve the corresponding equations for devices of both simple and complicated geometries, in the context of drug release systems. However, these models did not account for the mechanical properties degradation of these devices.

Polymer degradation is the first step of the erosion phenomenon. The complete erosion of the polymer is known to take substantially longer than the complete 
loss of tensile strength. During this first phase, aqueous solution penetrates the polymer, followed by hydrolytic degradation, converting these very long polymer chains into shorter water-soluble fragments, which can be regarded as a reverse polycondensation process. For example, PLA becomes soluble in water for molecular weight, $M_{n}$, below $\mathbf{0} 20.000$ (g/mol) (Zhang et al. 2008).

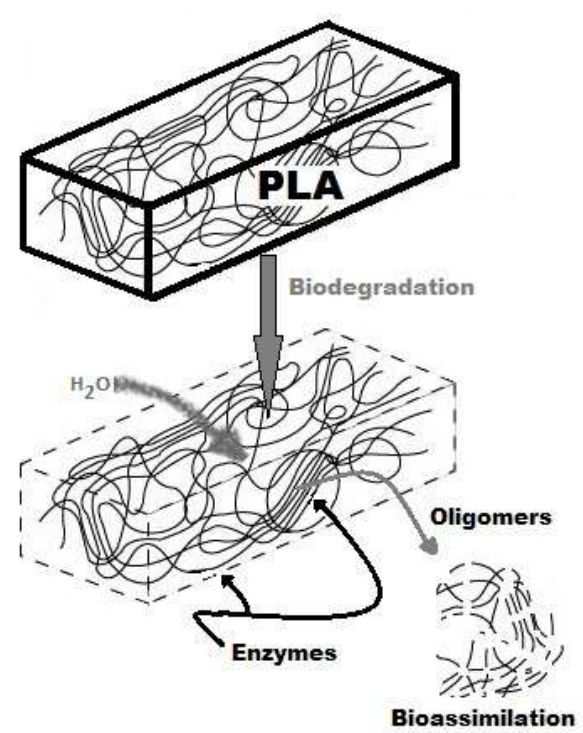

Fig. 1 - Scheme of erosion process

\section{Hydrolytic Damage}

Hydrolytic damage can be defined as the time-dependent cumulative irreversible damage due to the hydrolytic cleavage of polymeric molecules. After immersion of a biodegradable polymeric device in an aqueous medium, water uptake is the very first event that occurs, up to a saturation of water concentration that depends on the hydrophilicity of the polymer, its crystalline degree, the temperature, $\mathrm{pH}$ and flow of the media. This step is accompanied by volume expansion due to the fluid ingress, usually designated by swelling. The intrusion of water then triggers the chemical polymer degradation, leading to the scission of molecules and the creation of oligomers. The penetrating water rapidly creates a negative gradient of water concentrations from the surface to the centre as expected from a pure diffusion viewpoint. However, this gradient vanishes in a couple of hours or days, when the specimen saturates. Diffusion of small molecules like water is rather fast as compared with degradation that can take several months. Therefore, one can consider that hydrolysis of ester bonds starts homogeneously along the volume from the beginning, promoting bulk erosion ( $\mathrm{Li}$ et al. 1990). This assumption is 
very precise for small thickness devices, such as fibers or films. Water uptake can also lead to further recrystallization of the polymer. Water acts as a plasticizer, lowering the glass transition temperature and softening the material.

The water concentration $(w)$ along the thickness, and during incubation, is determined using Fick's equation:

$$
\frac{d w}{d t}=D_{1} \frac{\partial^{2} w}{\partial x^{2}}+D_{2} \frac{\partial^{2} w}{\partial y^{2}}+D_{3} \frac{\partial^{2} w}{\partial z^{2}}
$$

In the case of isotropic polymers, diffusion has no preferential direction, and $D_{1}=D_{2}=D_{3}=D$. The diffusion coefficient $D$ of the material can be determined by inverse parameterization, measuring the increase in weight due to moisture absorption during incubation, on samples with two different diameters. The amount of absorbed water is computed from:

$$
m_{w}=100 *\left(m_{w s}-m_{w r}\right) / m_{w r}
$$

where $m_{w r}$ and $m_{w s}$ are the weights of the specimen before and after absorption, respectively.

The macromolecular skeleton of many polymers comprises chemical bonds (e.g. polyethylene terephthalate PET, polybutylene terephthalate PBT, epoxies crosslinked by anhydrides, unsaturated polyesters, vinylesters, PLA, PGA, PCL and PHA's), such as ester groups, that can go through hydrolysis in the presence of water molecules, leading to chain scissions. In the case of polylactides, these scissions occur at the ester groups. Ester hydrolysis can be either acid or base catalyzed (Sykes 1975). In Figure 2, a scheme of the acid based hydrolysis mechanism, more common in PLA degradation, is presented. A general consequence of such a process is the lowering of the plastic flow ability of the polymer, thus causing the change of a ductile, tough behavior into a brittle one. If the behavior was initially brittle, there will be an increase in the brittleness. Each polymer molecule, with its own carboxylic and alcohol end groups, is broken in two, randomly in the middle at a given ester group. So, while the molecules are being splited by hydrolysis the number of carboxylic end groups will increase with degradation time (see Error! Reference source not found.).

Hydrolysis has traditionally been modeled using a first order kinetics equation based on the kinetic mechanism of hydrolysis, according to the Michaelis-Menten scheme (Bellenger et al. 1995). According to Farrar and Gilson (Farrar and Gilson 2002), the following first-order equation describes the hydrolytic process relative to the carboxyl end groups $(C)$, ester concentration $(E)$ and water concentration $(w)$ : 
$\frac{d C}{d t}=k E w C=u C$

where $u$ is the hydrolysis rate of the material, $k$ is the hydrolysis rate constant, assuming that $E$ and $w$ are constant in the early stages of the reaction. In addition, water is spread out uniformly in the sample volume (no diffusion control).

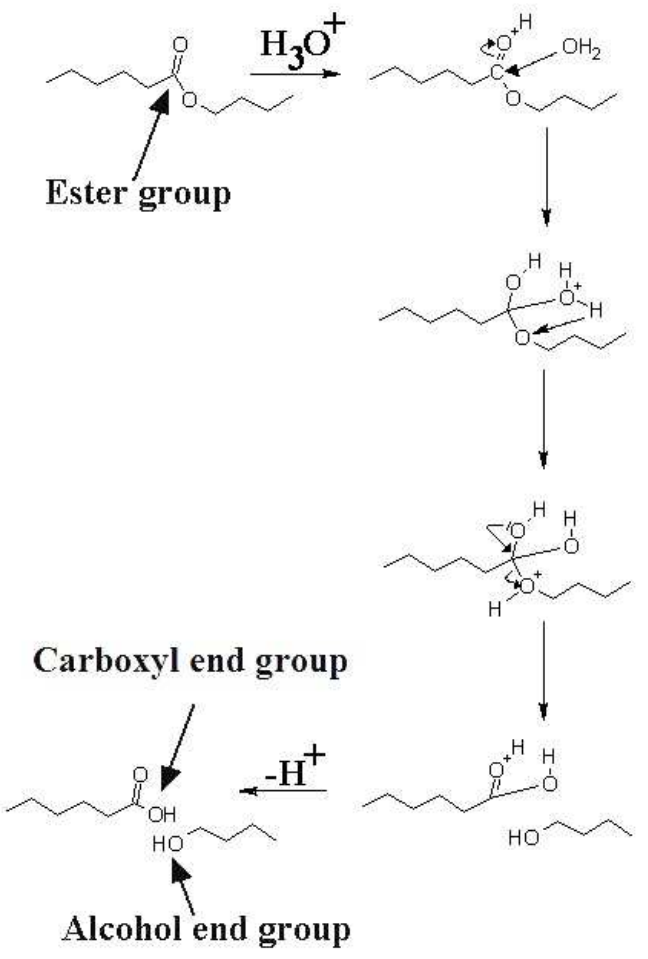

Fig. 2 - Acid catalyzed hydrolysis mechanism (Morrison and Boyd 1992)

Or using the scission number $n_{t}$ per mass unit, as presented in the literature (Bellenger et al. 1995), at time $t$ is given, and the initial concentration of carboxyl end groups $C_{0}$ is known, Equation 3 becomes:

$$
\frac{d n_{t}}{d t}=k E w\left(C_{0}+n_{t}\right)
$$

The experimental measurement of molecular weights allows the determination of $n_{t}$, and consequently the degradation rate constant: 
$n_{t}=\frac{1}{M_{n_{t}}}-\frac{1}{M_{n_{0}}}$

Using the molecular weight, and since the concentrations of carboxyl end groups are given by $C=1 / M_{n}$, the Equation 3 becomes:

$$
M_{n_{t}}=M_{n_{0}} e^{-u t}
$$

where $M_{n t}$ and $M_{n 0}$, are the number-average molecular weight, at a given time $t$ and initially at $t=0$, respectively. This equation leads to a relationship $M_{n}=f(t)$, and the result is in $\mathrm{g} / \mathrm{mol}$. However, in the design phase of a tissue engineering biodegradable device, it is important to predict the evolution of mechanical properties like tensile strength, instead of molecular weight. It has been shown (Ward 1983) that the fracture strength of a generic thermoplastic polymer can, in many cases, be related to $M_{n}$ through the empirical relationship:

$$
\sigma=\sigma_{\infty}-\frac{A}{M_{n_{t}}}=\sigma_{\infty}-\frac{A}{M_{n_{0}} e^{-u t}}
$$

where $\mathbf{\square}$ is the fracture strength, $\mathbf{\square} \mathbf{~ i s ~ t h e ~ f r a c t u r e ~ s t r e n g t h ~ a t ~ i n f i n i t e ~ m o l e c u l a r ~}$ weight, and $A$ is a material constant. Equation 7 provides a description of the time dependence of the material's mechanical strength, which is relevant in the design phase of a biodegradable device. Since this is an empirical equation, constant $A$ must be determined experimentally for each material. One can thus determine the limit strength of the device during the recovering of the tissue, $\sigma_{d}=f(t)$. When regenerating a tissue, the strength of the scaffold, $\sigma_{d}$, should be compatible to the strength of the new formed tissue $\sigma=\sigma(t)$. According to Farrar and Gilson (Farrar and Gilson 2002), the hydrolysis rate depends on the structure of the polymer, and is independent of its initial molecular weight. The Equation 7 is illustrated in Error! Reference source not found. 


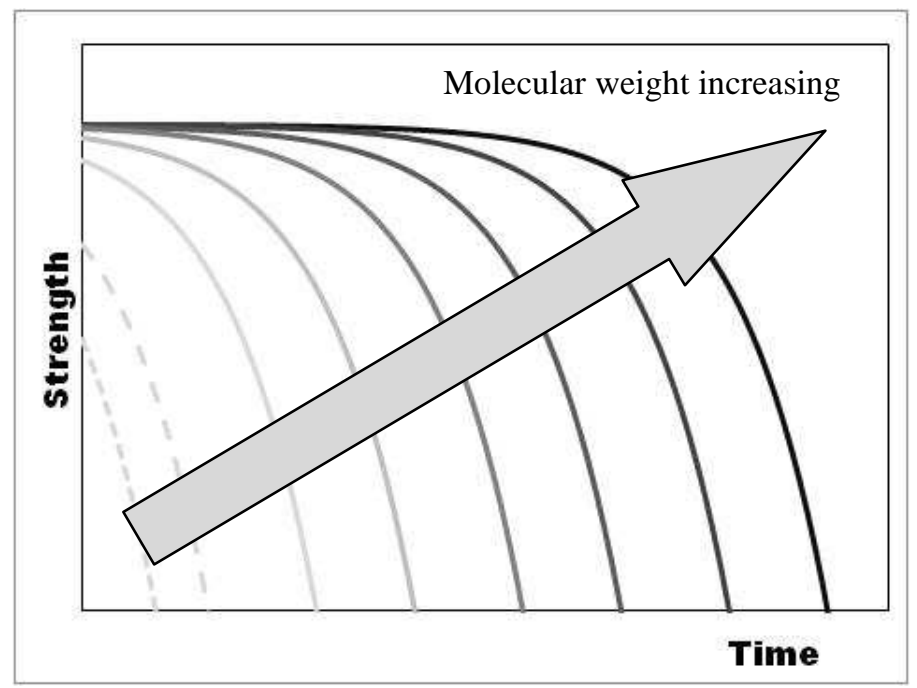

Fig. 3 - Tensile strength vs. time for different initial molecular weight (based on Farrar and Gilson 2002)

The storage or sterilization processes may pre-degrade the material, leading to reduction of degradation time and its initial mechanical strength, but the rate of degradation remains the same. To tune degradation time, specimens with different initial molecular weight can be created by gamma-irradiation starting from commercial materials available. Regrettably, this technique also reduces the initial mechanical properties of the materials.

The Equation 7 is not a very good model for tensile strength, except in the brittle failure regime for amorphous polymers or semi-crystalline polymers below their glass transition temperature. This is a common problem with highly ductile polymers. In these cases, it is more correct to use true values instead of nominal stress and strain, by assuming that the deformation occurs at constant volume (Ward 1983). In this case, the true area, $A$, is given by $A 0 /(1+\mathbf{0})$; where $A_{0}$ is the initial area and $\mathbf{q}$ is the nominal strain. This leads to the true stress being given by $(1+\mathbf{\square}) * \mathbf{\square} a$; where $\mathbf{D}_{a}$ is the apparent stress based on $A_{0}$.

As it will be shown in the next sections, strength follows the same trend as the molecular weight:

$\sigma_{\mathrm{t}}=\sigma_{0} * \mathrm{e}^{-\mathrm{ut}}$

The hydrolytic damage, defined by the ratio between the initial strength of the virgin material and the current strength, after a certain degradation time, is:

$d_{h}=1-\frac{\sigma_{t}}{\sigma_{0}}=1-e^{-u t}=1-e^{-k E w t}$ 
So the hydrolytic damage depends on the hydrolysis kinetic constant, $k$, the concentrations of ester groups, $E$, the water concentration in the polymer matrix, $w$, and the degradation time $t$. The hydrolysis kinetic constant, $k$, is a thermodynamic quantity associated with the probability of molecular scission, and it depends on temperature, load applied to the material and $\mathrm{pH}$ of the aqueous media. The $\mathrm{pH}$ of the aqueous medium also affects the hydrolysis reaction rates (Kirby 1972). Tsuji et al. studied the hydrolysis of PLLA films at $37{ }^{\circ} \mathrm{C}$ in alkaline solution $(\mathrm{pH} \mathrm{12})$ (Tsuji and Ikada 1998), acid solution ( $\mathrm{pH}$ 2.0) (Tsuji and Nakahara 2001) and phosphate-buffered solutions ( $\mathrm{pH}$ 7.4) (Tsuji and Ikada 2000). In the human body, $\mathrm{pH}$ can be considered constant, kept by the organism at a homeostatic value.

Temperature will augment diffusion due to increased molecular flexibility, but it will also amplify the hydrolysis rate, due to excitement of the molecules that it will raise the probability of bond scissions. Also, in the human body, temperature is kept constant at the homeostatic value of around $37^{\circ} \mathrm{C}$. The influence of the mechanical environment in the hydrolysis rate was also reported (Miller and Williams 1984; Chu 1985). Loaded fibers degrade faster than unloaded ones, and the magnitude of degradation depends on the level of applied stress and the incubation time. Similarly to temperature, stress also increases the probability of bond scissions. In most applications, the material is submitted to a stress state. When the stress state remains constant during degradation, the hydrolysis rate must be determined for that particular load case. If any variation were to occur in the stress state, temperature, or environment, $k$ would no longer be constant.

In this example, homogeneous degradation with instant diffusion, the hydrolysis rate, $u$, is constant, and damage only depends on degradation time. Although, these considerations are valid in the majority of the cases, in some cases, the hydrolysis rate cannot be considered constant. In brief, the hydrolysis rate of the material $(u)$ should be determined experimentally in accordance to the degradation environment of the application. In the characterization section, an example degradation rate determination will be presented.

\section{Further refinements of degradation models}

In a complex organism, several substances are responsible for degradation. More precise models can include each one of these substances. Bioprocess models are often restricted to the evolution of macroscopic species involved in a reaction scheme (Bastin and Dochain 1990). Such a reaction scheme describes the main phenomena occurring in the culture and is typically built of a reduced number of irreversible reactions involving macroscopic species. For each enzyme and water, its hydrolytic effect is usually modeled, using a first order differential equation, with different hydrolytic constant rates and concentrations that must be known. The model used is formally based on the kinetic mechanism of enzymatic hydrolysis according to the Michaelis-Menten scheme (Tzafriri et al. 2002): 


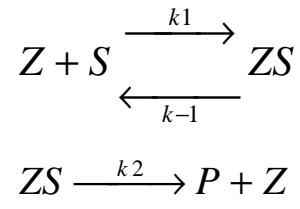

where $Z$ and $S$ represent the enzyme and substrate polymer, respectively and $Z S$ is the enzyme/substrate complex, $P$ is used to denote the hydrolysis reaction products, $k 1, k-1$ and $k 2$ are rate parameters. $k l$ describes the diffusion and adsorption of the enzyme onto the substrate polymer, $k-1$ the dissociation of the ZS complex without degradation (in general, equal to zero) (Tzafriri et al. 2002) and $k 2$ the degradation process. The degradation is mostly assumed to be the rate-limiting step, because equilibrium in adsorption is much faster compared to degradation.

In order to perform computer simulation based on these models, the equations can be discretized using the mixed finite element method for the space and an implicit scheme for the time. Having determined the concentration of the carboxylic end groups at the $\mathrm{n}^{\text {th }}$ discrete time point, the degradation equation can be solved and it can proceed to the next time step (or increment).

When a process is composed of a sequence of reactions, the overall rate is determined by the slowest reaction, named the rate-limiting step (Hill 1977). Klyosov and Rabinowitch (Klyosov and Rabinowitch 1980) reported that the rate limiting step may change between the beginning of the reaction and after a certain degree of substrate conversion.

The degree of crystallinity may also be a crucial factor, since hydrolysis occurs mainly in the amorphous domains. Water and enzymes degrade the more accessible amorphous region, but are unable to attack the less accessible crystalline portions. The water permeability along the crystalline region is much smaller than amorphous region. The observed increase in percentage of the crystalline phase is explained by the faster degradation that occurs in the amorphous region. Polymers with low crystallinity showed increased hydrolysis rates (Seretoudi et al. 2002; Shen-Guo and Bo 1992). As the crystallinity increases steadily throughout the reaction, substrate becomes increasingly resistant to further hydrolysis (Fan and Lee 1983; Fan et al. 1980), therefore affecting the kinetics of the process (Kennedy and Melo 1990; Walker and Wilson 1991). To model this phenomenon, knowing the initial crystalline degree, two different rates can be considered for both phases, and two different hydrolytic damage values should be calculated and added according to the volume fractions. The crystallinity of copolymers $(X \%)$ can be determined by dividing the observed heat of fusion in a DSC (Differential Scanning Calorimetry) test, by the theoretical value for perfectly crystalline polymer according to:

$$
X \%=\frac{\Delta h_{m}}{\Delta h_{m}^{0}}
$$


Crystallinity also affects the mechanical properties of materials. Their glass transition temperature is lowered due to water uptake, which can lead to recrystallization of the polymer. Hence, material processing and storage conditions have a great influence on mechanical and degradation properties (Saha and Tsuji 2009).

\section{Surface vs. Bulk Erosion}

All degradable polymers share the property of eroding upon degradation. The water ingress triggers the chemical polymer degradation leading to the creation of oligomers and monomers. Progressive degradation changes the microstructure of the bulk through the formation of pore via which oligomers are released. Concomitantly, the $\mathrm{pH}$ inside pores begins to be controlled by degradation products, which typically have some acid-base functionality. Finally, oligomers and monomers are released, leading to the weight loss of polymer devices. The distinction made between surface (or heterogeneous) and bulk (or homogeneous) eroding materials is used to classify degradable polymers.

Different types of erosion are illustrated in Figure 4. In Figure $4 \mathrm{c}$ ), there is a typical case of homogeneous or bulk erosion without autocatalysis, in which diffusion occurs instantaneously. Hence, the decrease in molecular weight, the reduction in mechanical properties, and the loss of mass occur simultaneously throughout the entire specimen. Polymers containing, ether, amide or ester groups, such as PLA, PGA, PCL, polyamide, proteins, and cellulose (and its derivatives), generally exhibit this type of erosion (Pitt et al. 1982).

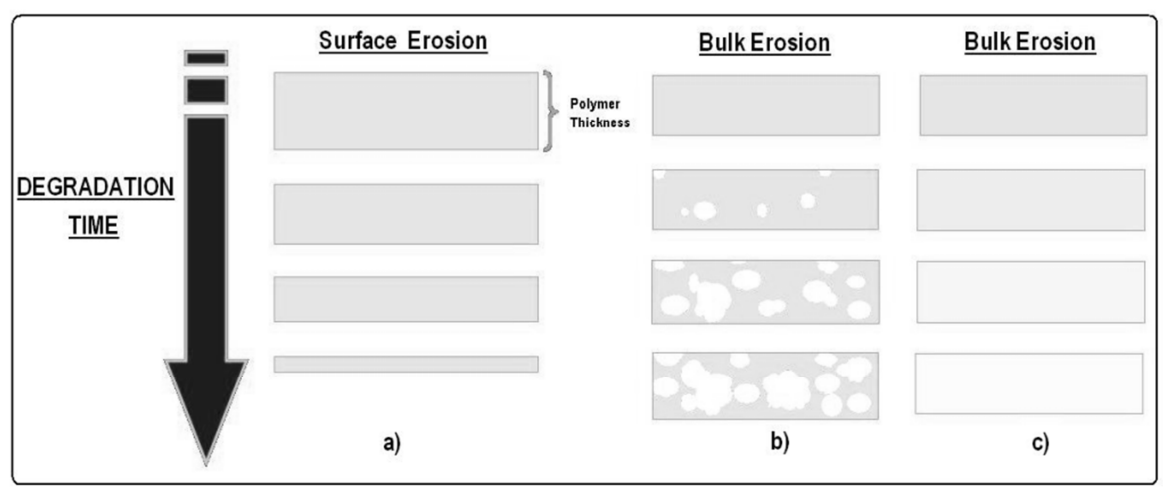

Fig. 4 - Schematic illustration of three types of erosion phenomenon: (a) surface erosion, (b) bulk erosion with autocatalysis, (c) bulk erosion without autocatalysis (based on von Burkersroda et al. 2002)

One other type is heterogeneous or surface erosion (Error! Reference source not found.a), in which hydrolysis occurs in the region near the surface, whereas the 
bulk material is only slightly or not hydrolyzed at all. As the surface is eroded and removed, the hydrolysis front moves through the material core. In this case, in which diffusion is very slow compared to hydrolysis, one must use Equation 1 to calculate water concentration $w(t, x)$ at any instant $t$ through the volume, before using Equations 8 or 9 . The rate of boundary movement is very often nearly constant (Lyu et al. 2005). Surface eroding polymers have a greater ability to achieve zero-order release kinetics, i.e. a state at which the rate of an enzyme reaction is independent of the concentration of the substrate. Therefore, they are ideal candidates for developing devices able to deliver substances (Nair and Laurencin 2007) such as drugs, growth factors, etc. Polymers such as poly(ortho ester)s (POEs), PAHs, and some polycarbonates tend to undergo surface erosion (von Burkersroda et al. 2002). Also enzymatic erosion fits on this last type of erosion, since enzymes are unable to diffuse and present a raised hydrolysis kinetic constant $k$. In the presence of enzymes, heterogeneous hydrolytic damage can be modeled, considering a high hydrolysis kinetic constant $k$ and a diffusion coefficient $D$ close to zero. This damage should then be added to the damage due to water, either homogeneous or heterogeneous.

Surface and bulk erosion are ideal cases to which most polymers cannot be unequivocally assigned. Hence, two major processes have an impact on the erosion kinetics:

1. the diffusion of water into the polymer bulk, and

2. the hydrolytic degradation of the polymer backbone.

It is possible to define the characteristic time of hydrolysis, $\mathbf{a}_{H}$, as the inverse of hydrolysis rate (Göpferich 1996):

$$
\tau_{H}=\frac{1}{k E w}=\frac{1}{u}
$$

If $D$ is the diffusion coefficient of water in the polymer and $L$ is the sample thickness, it is also defined a characteristic time of diffusion, $\mathbf{a}_{D}$ (Göpferich 1996):

$$
\tau_{D}=\frac{L^{2}}{D}
$$

For assuming that the sample surface is large enough so that it can be neglected the edge effects. When $\mathbf{a}_{H} \gg \mathbf{q}_{D}$, water reaches the core of the material before it reacts, and the degradation starts homogenously. When $\mathbf{D}_{H}<\mathbf{q}_{D}$, water reacts totally in the superficial layer and will never reach the core of the material. The degradation starts heterogeneously through the volume. In these cases, a higher surface to volume ratio induces a faster degradation. So, in heterogeneous degradation fibers of smaller diameter will have, in average, a higher hydrolysis rate $u$ than the larger diameter fibers. Critical conditions are defined when $\mathbf{D}_{D}=\mathbf{D}_{H}$. In this critical condition, the critical thickness can be defined as (Göpferich 1996): 
$L_{c}=\sqrt{\frac{D}{u}}$

This critical thickness ranges from tens of micrometers for PAH's to a few centimeters for polyesters (Göpferich 1996). If the specimen thickness is larger than this critical size, the specimen undergoes surface erosion. Otherwise, it undergoes bulk erosion. Since diffusion and hydrolysis depend on temperature, $\mathrm{pH}$ of the aqueous media, etc., the critical thickness will depend on those parameters. According to Göpferich's point of view (Göpferich 1996), all the water-insoluble degradable polymers could undergo surface erosion or bulk erosion at different conditions. In conclusion, the way a polymer matrix erodes depends on the diffusivity of water inside the matrix, the hydrolysis rate of the polymer's functional groups and the matrix dimensions. It should be noted that if laboratory experiments are carried out on samples with thickness lower than the critical value, they will not necessarily model thicker samples.

One factor that complicates the erosion is the autocatalytic hydrolysis reaction (Siparsky et al. 1998). The hydrolytic degradation of aliphatic polyesters derived from lactic and glycolic acids (PLA/GA polymers) has been previously shown to proceed heterogeneously in the case of large size devices, the rate of degradation being greater inside than at the surface (Li et al. 1990; Vert et al. 1991; Grizzi et al. 1993). This was observed both in vitro (Grizzi et al. 1995) and in vivo (Therin et al. 1992). For example, a thick plate of PLA erodes faster than a thinner one made of the same polymer (Grizzi et al. 1995). This occurs due to retention of the oligomeric hydrolysis products within the material, unable to diffuse out if the material is very thick. These oligomeric reaction products are carboxylic acids, causing a decrease in $\mathrm{pH}$ and increased hydrofilicity in the core of the material, therefore accelerating locally the degradation (Göpferich 1996) due to a local increase of hydrolysis kinetic constant, $k$.

As degradation proceeds, soluble oligomers which are close to the surface can leach out, whereas those which are located well inside the matrix remain entrapped and fully contribute to the autocatalytic effect. This difference of concentration in acidic groups, results in the formation of a skin composed of less degraded polymer. The thickness of this skin depends on many factors such as the diffusion rates of each involved species and the rate of ester bond cleavage. As can be seen in Error! Reference source not found.b), hollow structures are formed as a consequence (Grizzi et al. 1995). A more complex model, with more parameters, is necessary to describe this phenomenon. This implies an extensive experimental characterization. However, this hollow formation occurs in the late stages of erosion, when molecular weight becomes greatly reduced. The models presented in the following section, to describe strength decrease and stress-strain plot evolution during degradation are only valid for the initial phase of erosion, i.e. for hydrolytic damage of about $50 \%$. Hence, these models neglect the hollow formation effect, since this phenomenon may be neglected during the first $50 \%$ of 
strength loss, i.e. the mass loss and oligomer diffusion are neglected (as will be showed in the following sections).

Some authors claim that the local raise of degradation rate can also be explained by the local increase of hydrophilicity. The hydrophilicity involves the build-up of acid and alcohol groups, much more hydrophilic than the initial ester group (Van Krevelen 1976). An increasing water equilibrium concentration with time can thus be expected. It is quite simple to solve the problem, as Bellenger et al. (Bellenger et al. 1995) have shown, if it were considered, with Van Krevelen (Van Krevelen 1976), that the water equilibrium concentration is an additive function, thus, $w=b$ $+a n t$, and:

$\frac{d C}{d t}=\frac{d n_{t}}{d t}=k E\left(C_{0}+n_{t}\right)\left(b+a n_{t}\right)$

Solving the Equation 15, as demonstrated by Bellenger et al. (Bellenger et al. 1995), leads to:

$n_{r}=C_{0}\left[\frac{b-a C_{0}}{b e^{-k E\left(b-a C_{0}\right) t}-a C_{0}}-1\right]$

were $a$ and $b$ are material parameters. Accordingly to Bellenger et al. (Bellenger et al. 1995), this equation gives a good quantitative description of the autoaccelerated character of the degradation. If the auto-accelerated character is not due to increasing hydrophilicity, it is probably because its origin is in the hydrolysis mechanism. Alcohol groups and especially acid groups coming from the first degradation steps can catalyze later hydrolysis reactions.

\section{Tuning hydrolytic rate according to scaffold requirements}

To control the hydrolytic rate, in order to match the dimensioning requests during all the healing process, the project designer can combine different materials with different hydrolytic rates. A wide range of degradation times and mechanical properties are possible, using different commercial available materials and varying dimensions and 3D architecture. One possible approach is the composite concept, making use of the broad range of material properties to construct a multilayer device, each layer possessing its own degradation rate. The mixture law may also be applied to hydrolytic rate, assuming homogenous degradation:

$u_{c}=u_{1} * V_{1}+u_{2} * V_{2}+\ldots+u_{i} * V_{i}+\ldots+u_{n} * V_{n}=\sum_{i=1}^{n} u_{n} * V_{n}$ 
where $V_{i}$ are the volume fractions of each material. Another way to control the hydrolytic rate constant of materials is by block copolymerization or blending with other biodegradable polymers, having different hydrolytic rate constants. Copolymers of several lactides or lactones can be synthesized by ring opening polymerization, resulting in high molecular weight polyesters (Endo et al. 1987). The mixture of different polymers to produce blends, with controlled hydrolytic rate and mechanical properties can be performed in two ways: mixing the melted polymers, or mixing polymers solutions using a common solvent. However, the miscibility is limited, depending on polymers used and its volume fractions. The observation of two glass transition temperatures is a common way to evaluate immiscibility of the blends. In blends formulations with poor miscibility, the mixture of polymer solutions is preferable. Unfortunately, this solution implies the use of solvents, which have negative environmental effects. The different strategies to control degradation rate are represented in Figure 5.

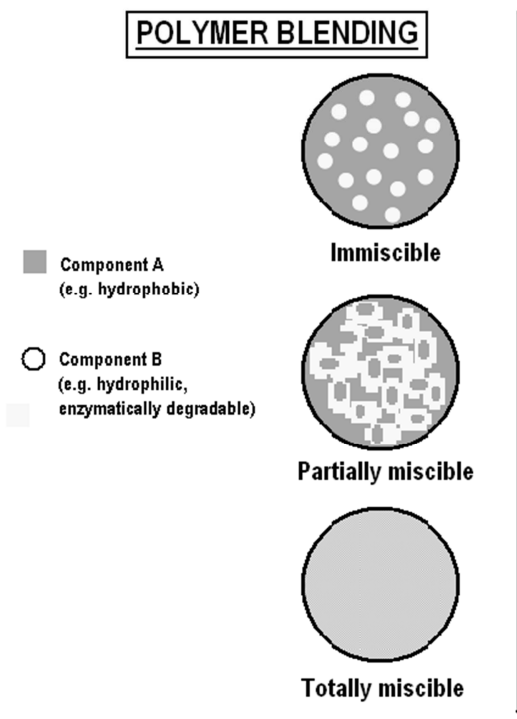

\section{CO-POLYMERISATION}

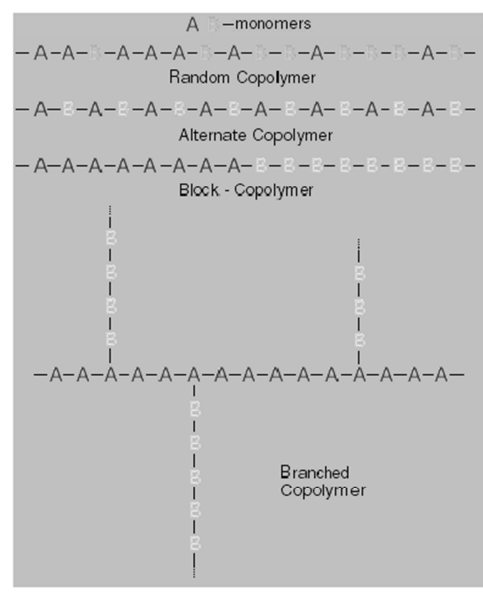

Fig. 5 - Strategies to control degradation rate of biodegradable polymers

\section{Characterization of degradation rate}

The aim of this section is to demonstrate an example of experimental procedure to analyze erosion and degradation. Weight, strength and molecular weight evolutions were determined during degradation of polymers. At the end of this procedure, it was possible to determine the degradation rate $u$ of the biodegradable material. In this example, and in the following sections, the material used is a blend 
of PLA-PCL (90:10). Two fiber dimensions were used (0.15 and $0.4 \mathrm{~mm})$. Samples were placed in tubes and submitted to different degradation stages, under PBS (Phosphate Buffer Solution) at $37^{\circ} \mathrm{C}$. The duration of stages was previously determined, according to the supplier durability claims, until a maximum of 7 months. At the end of each degradation stage, $\mathrm{pH}$ of the media was measured, then test pieces were weighted after and before drying, further submitted to tensile tests and finally to GPC (Gel Permeation Chromatography) to measure molecular weight. The initial $\mathrm{pH}$ of the PBS solution was 8 (eight) and did not change significantly during degradation. As can be seen in Figure 6, PLA-PCL has become brittle only after 16 weeks, lost its plasticity region, and strength has progressively decreased. The almost constant slope of the linear elastic stage indicates that no significant variation in Young modulus occurred during degradation.

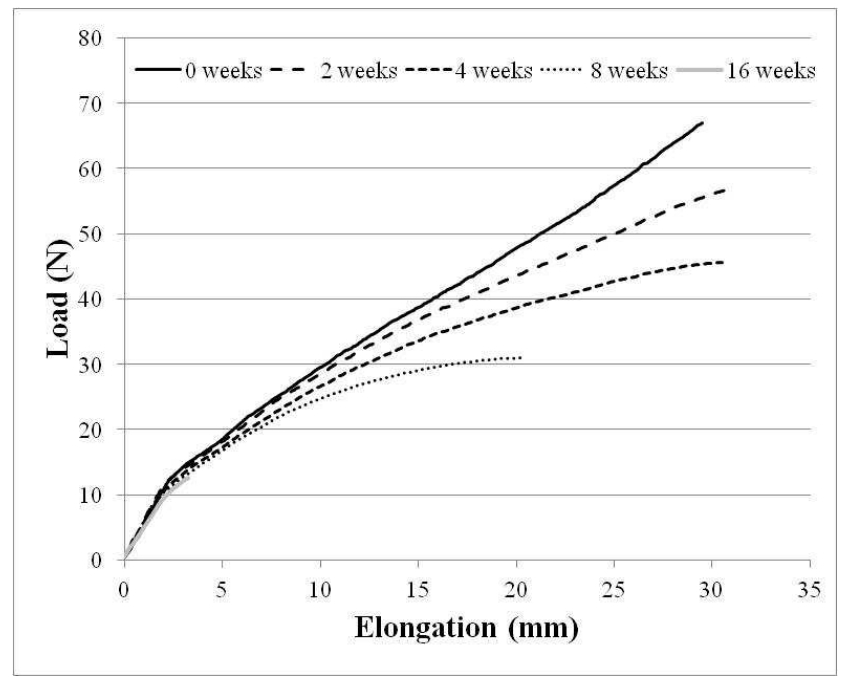

Fig. 6 - Tensile test results for different degradation time of PLA-PCL fibers $(400 \mu \mathrm{m})$ under PBS

As can be seen in Figure 7, while in the first 16 weeks the fiber only looses $10 \%$ of mass, it presents $80 \%$ of strength loss. For these PLA-PCL fibers, no significant differences were observed among the different dimensions tested, either in terms of strength and molecular weight evolutions during degradation (see Figure 8). One can conclude that, in the present case, water diffusion can be assumed instantaneous and that hydrolysis takes place homogenously throughout the samples (bulk degradation without autocatalysis) (Auras et al. 2004). For highly heterogeneous degradation, the rate will not be globally similar, independently of dimensions, and the water concentration will locally depend on the position and time. 


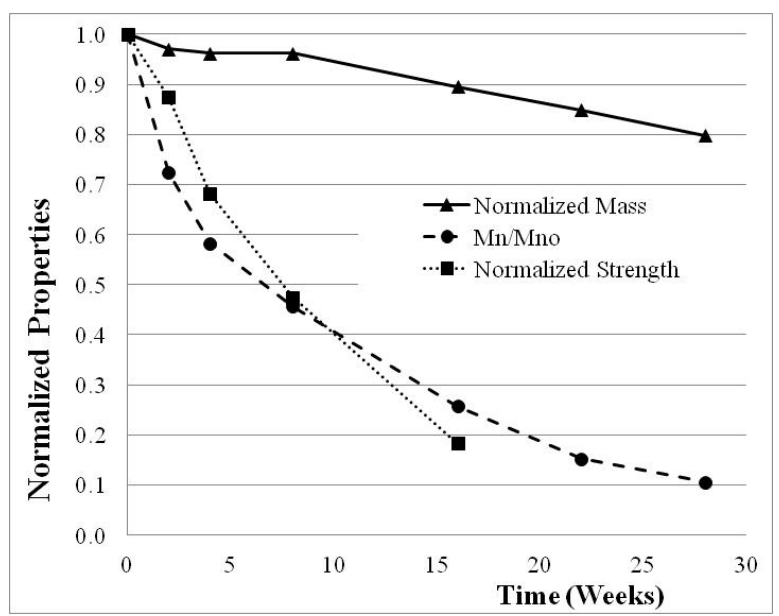

Fig. 7 - Normalized: a) mass, b) molecular weight and strength, for different degradation time of PLA-PCL fibers, of $400 \mu \mathrm{m}$, under PBS

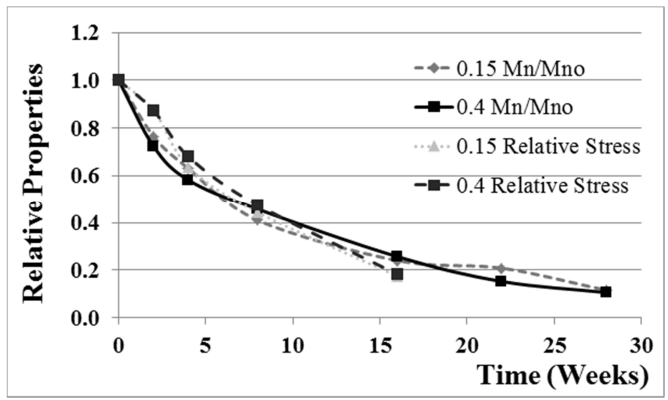

Fig. 8 - Normalized: a) strength and b) molecular weight, for different degradation time of PLA-PCL fibers, of $150 \mu \mathrm{m}$ and $400 \mu \mathrm{m}$, under PBS

From Figure 9, one can see that the measured strength follows the same trend as the molecular weight, in a semi-logarithmic representation. The slope of this linear fitting, that includes all experimental results normalized to the initial value and in semi-logarithmic scale, represents the degradation rate. Instead of Equation 8, a relationship similar to the one obtained for the molecular weight, Equation 7, can be used,

$$
\sigma=\sigma_{0} e^{-u_{s} t}
$$

where $u_{s}$ is the strength decrease rate of the material. This parameter, $u_{s}$, seems to be directly related to the molecular weight decrease rate of the material, $u_{m}$, as can be seen in Figure 9 and in Table 1. This same trend can be found in the degrada- 
tion results of other previous works, such as the one by Meek et al. (Meek et al. 2004), with PDLA-PCL. This can therefore provide a strategy to obtain a design failure criterion for the evolution of the limit strength of the device during the degradation process, $\sigma=f(t)$.

Table 1 - Degradation rate of PLA-PCL under PBS, determined by measuring strength and molecular weight evolution for different degradation time

\begin{tabular}{|c|c|c|c|c|}
\hline & $\operatorname{Ln}\left(\mathbf{\square} / \mathbf{\square}_{o}\right)=-u_{s} t$ & $\mathrm{R}$ & $\operatorname{Ln}\left(M_{n} / M_{o}\right)=-u_{m} t$ & $\mathrm{R}$ \\
\hline$u$ & 0.103 & 0.996 & 0.0841 & 0.989 \\
\hline
\end{tabular}

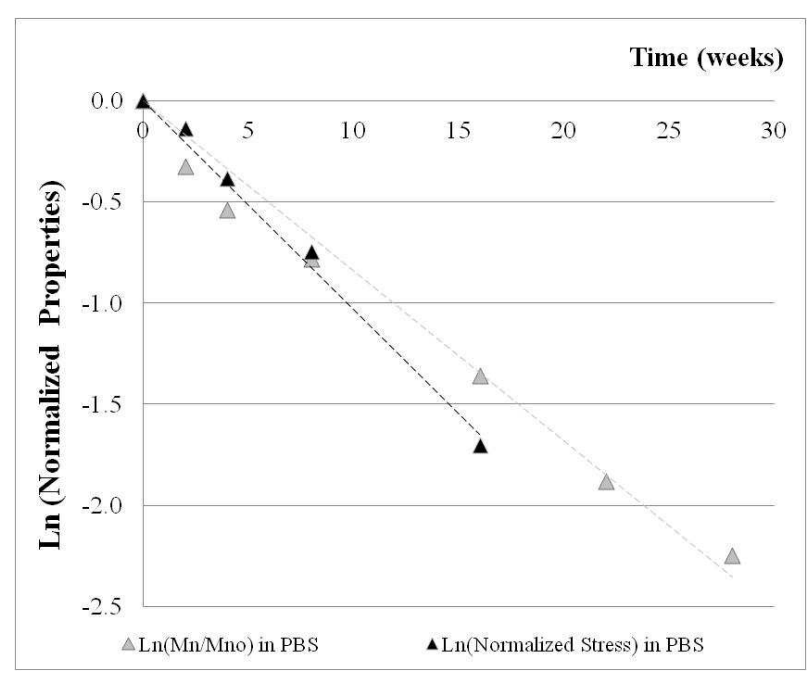

Fig. 9 - Normalized strength and normalized molecular weight evolution for different degradation time of PLA-PCL fibers of $400 \mu \mathrm{m}$ under PBS

\section{Constitutive models to simulate mechanical behavior during hydrolytic degradation}

Whenever loading conditions are simple and the desired lifetime of mechanical support is known, a "trial and error" approach may be sufficient to design reasonable reliable devices. In more complex situations, scaffolds designers can use numerical approaches to define the material formulation and geometry that will satisfy the immediate needs of symptomatic relief. In these cases, they can use constitutive models supplied in the commercial packages of Finite Element Method (FEM) modeling, to simulate the mechanical behavior of a device in the most severe condition, based on the initial material mechanical properties. However, the 
lack of design tools to predict long term behavior has limited the application of biodegradable materials.

A constitutive model for a mechanical analysis is a relationship between the response of a body (for example, strain state) and the stress state due to the forces acting on the body, which can include the environmental effects. A wide variety of material behaviors are described with a few different classes of constitutive equations. Mechanical properties of biodegradable plastics are commonly assessed within the scope of linearized elasticity, despite the clear evidence that they can undergo large strains before breaking. Due to the nonlinear nature of the stress $v s$. strain plot, the classical linear elastic model is clearly not valid for large strains simulation. Other plasticity or hyperelastic models are required to model those situations. Hence, given the nature of biodegradable polymers, classical models such as the Neo-Hookean and Mooney-Rivlin models, for incompressible hyperelastic materials, may be used to predict mechanical behavior until rupture of nondegraded PLA (Garlotta 2001; Lunt 1998). A single-order, isotropic Ogden material hyperelastic model was also used (Krynauw et al. 2011) to simulate the mechanical behavior evolution during degradation of a polyester-urethane scaffold. These models are useful to model the toughness of materials with this type of mechanical behavior. For these materials, the work assumption implies the existence of a scalar field, the stored energy function $W$, which is a function of the deformation gradient $F$. The stored energy function, $W$, can also be represented as a function of the right Cauchy-Green deformation tensor invariants. In general, the strain energy density for an isotropic, incompressible, hyperelastic material is determined by two invariants. The first and second invariants in uniaxial tension are given by:

$$
\begin{aligned}
& I_{C}=\lambda^{2}+\frac{2}{\lambda} \\
& I_{C}=\frac{1}{\lambda^{2}}+2 \lambda
\end{aligned}
$$

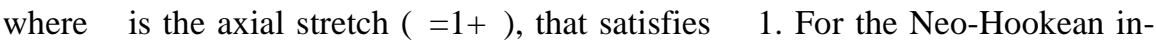
compressible hyperelastic solid, the stored energy function is given by:

$W=\frac{\mu_{1}}{2}\left(I_{C}-3\right)$

where $\mu_{1}>0$ is the material property, usually called the shear modulus. An extension of this model is the Mooney-Rivlin incompressible hyperelastic solid, which stored energy function has the form:

$W=\frac{\mu_{1}}{2}\left(I_{C}-3\right)+\frac{\mu_{2}}{2}\left(I I_{C}-3\right)$ 
with two material properties $\mu_{1}$ and $\mu_{2}>0$. Higher order stored energy functions may be considered to describe the experimental data, such as a reduced $2^{\text {nd }}$ order stored energy function, that includes a mixed term with both invariants of the right Cauchy-Green stretch tensor and an extra material constant $\mu$, which the stored energy function has the form:

$W=\frac{\mu_{1}}{2}\left(I_{C}-3\right)+\frac{\mu_{2}}{2}\left(I I_{C}-3\right)+\frac{\mu_{3}}{6}\left(I_{C}-3\right)\left(I I_{C}-3\right)$

Considering the equations above, the axial nominal stress for the three models, Neo-Hookean $\left(\mathbf{a}^{N H}\right)$, Mooney-Rivlin $\left(\mathbf{\square}^{M R}\right)$ and reduced second order $\left(\mathbf{a}^{2 n d ~ r e d}\right)$, will be given by:

$$
\begin{aligned}
& \sigma^{N H}=\mu_{1}\left(\lambda-\frac{1}{\lambda^{2}}\right) \\
& \sigma^{M R}=\mu_{1}\left(\lambda-\frac{1}{\lambda^{2}}\right)+\mu_{2}\left(1-\frac{1}{\lambda^{3}}\right) \\
& \sigma^{2^{n d} r e d}=\left(\mu_{1}-\mu_{3}\right)\left(\lambda-\frac{1}{\lambda^{2}}\right)+\left(\mu_{2}-\mu_{3}\right)\left(1-\frac{1}{\lambda^{3}}\right)+\mu_{3}\left(\lambda^{2}-\frac{1}{\lambda^{4}}\right)
\end{aligned}
$$

According to Soares et al. (Soares et al. 2010), the model constitutive material parameters depend on degradation time. The material parameters are considered to be material functions of degradation damage instead of material constants. For fibers of a blend of PLA-PCL (90:10), it was determined that only the first material parameter $\mu_{l}$ varies linearly with hydrolytic damage (as defined in Equation 9) (Vieira et al. 2011b).

From Error! Reference source not found., one can see that the hyperelastic material models fit well the measured storage energy, for all the degradation steps up to 8 weeks (about $50 \%$ of damage). The experimental data of storage energy was calculated by measuring the area (i.e., by taking the integral) underneath the stress-strain curve, from initial stretch (one) to the desired stretch level (no compression behavior was accessed). The Neo-Hookean model was the less accurate. However, it never violates the $2^{\text {nd }}$ Law of Thermodynamics, which imposes that every material parameters $\mu_{i}$ must be positive. The material parameters were calculated by inverse parameterization based on the experimental data. The results are presented in Table 2.

If the last degradation stage is discarded, then the material model parameter, $\mu_{1}$, varies linearly with the hydrolytic damage, as proposed by Soares et al. (Soares et al. 2010). The proposed approach, which admits that only the first material parameter changes with hydrolytic damage, $\mu_{l}(d)$, according to the linear regressions (see Figure 11), allows a good description of the mechanical behavior evolution, based on Equations 24, 25 or 26. Moreover the ultimate stress, which is the failure criterion used, can be defined by Equation 18 . 

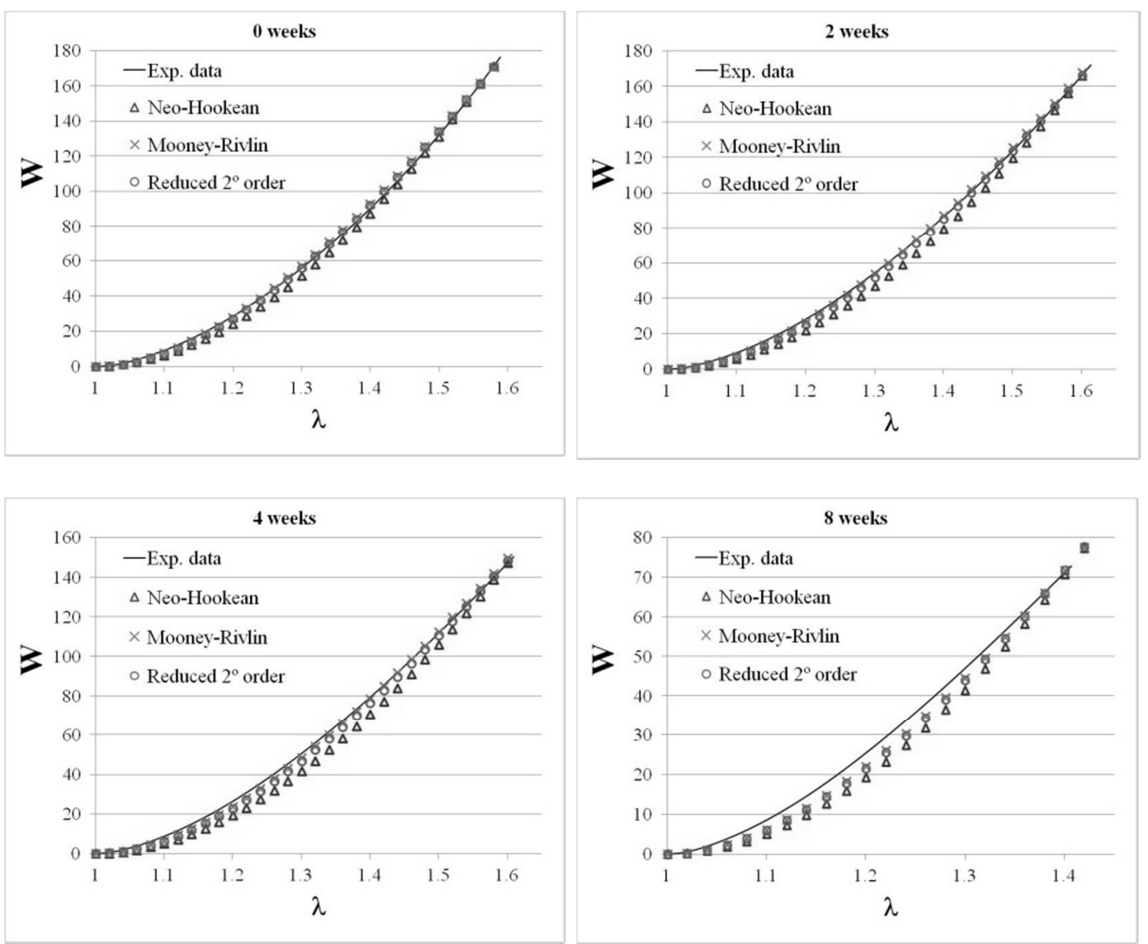

Fig. 10 - Storage energy vs. axial stretch for $0,2,4$ and 8 weeks of degradation (Vieira et al. 2011b)

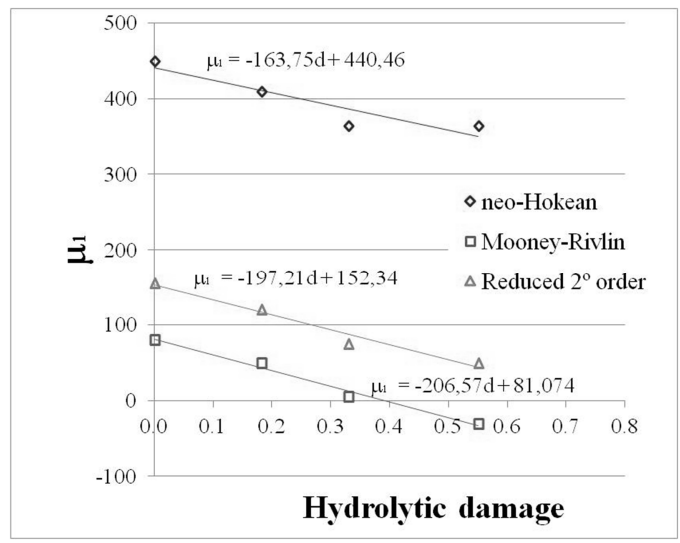

Fig. 11 - Evolution of the material parameter, $\mu_{1}$, for each model, considering different degradation time (Vieira et al. 2011b) 
Table 2 - Material models parameters for different degradation time (Vieira et al. 2011b)

\begin{tabular}{lccccc}
\hline Material Models & Weeks & $\mathrm{D}$ & $\mu_{1}$ & $\mu_{2}$ & $\mu_{3}$ \\
\hline \multirow{4}{*}{ Neo-Hookean } & 0 & 0.00 & 450 & & \\
& 2 & 0.18 & 410 & & \\
& 4 & 0.33 & 364 & - & - \\
& 8 & 0.55 & 364 & & \\
Mooney-Rivlin & 16 & 0.80 & 630 & & \\
& 0 & 0.00 & 80 & & \\
& 2 & 0.18 & 50 & & \\
& 4 & 0.33 & 5 & 500 & - \\
& 8 & 0.55 & -30 & & \\
$2^{\text {2d }}$ reduced order & 16 & 0.80 & 150 & & \\
& 0 & 0.00 & 155 & & \\
& 2 & 0.18 & 120 & & \\
& 4 & 0.33 & 75 & 400 & -1 \\
& 8 & 0.55 & 50 & & \\
\hline
\end{tabular}

From Error! Reference source not found., one can see that the hyper elastic material models allowed a reasonable approximation of the tensile test results, i.e. stress vs. strain. However, the constitutive models are unable to describe precisely the initial elastic phase of the stress-strain plot, where the stiffness remains barely constant. This explains why the material model parameter, $\mu_{l}$, increases sharply in the last degradation stage (16 weeks) for all three models, because the inverse parameterization was based on the experimental data that mostly comprehends elastic deformation.

Tensile strength evolution can be determined during degradation in test specimens of PLA-PCL fibers or other elements with small thickness. This is possible since hydrolytic reaction is the limiting step of the overall degradation process. Diffusion may be neglected in these cases, and hydrolysis may be considered to take place homogeneously within the sample volume.

These constitutive models are available in commercial FEM software packages, but they are not linked to failure criterion. Thus, a new approach is proposed in which constitutive equations can be implemented in commercial FEM software packages like ABAQUS ${ }^{\mathrm{TM}}$, by changing the material parameter as function of hydrolytic damage or degradation time, and associated to the failure criterion implemented by a User Material (UMAT) subroutine, as well as PYTHON language. An example of this approach is given in the following section, for a simple geometry of a fiber. 

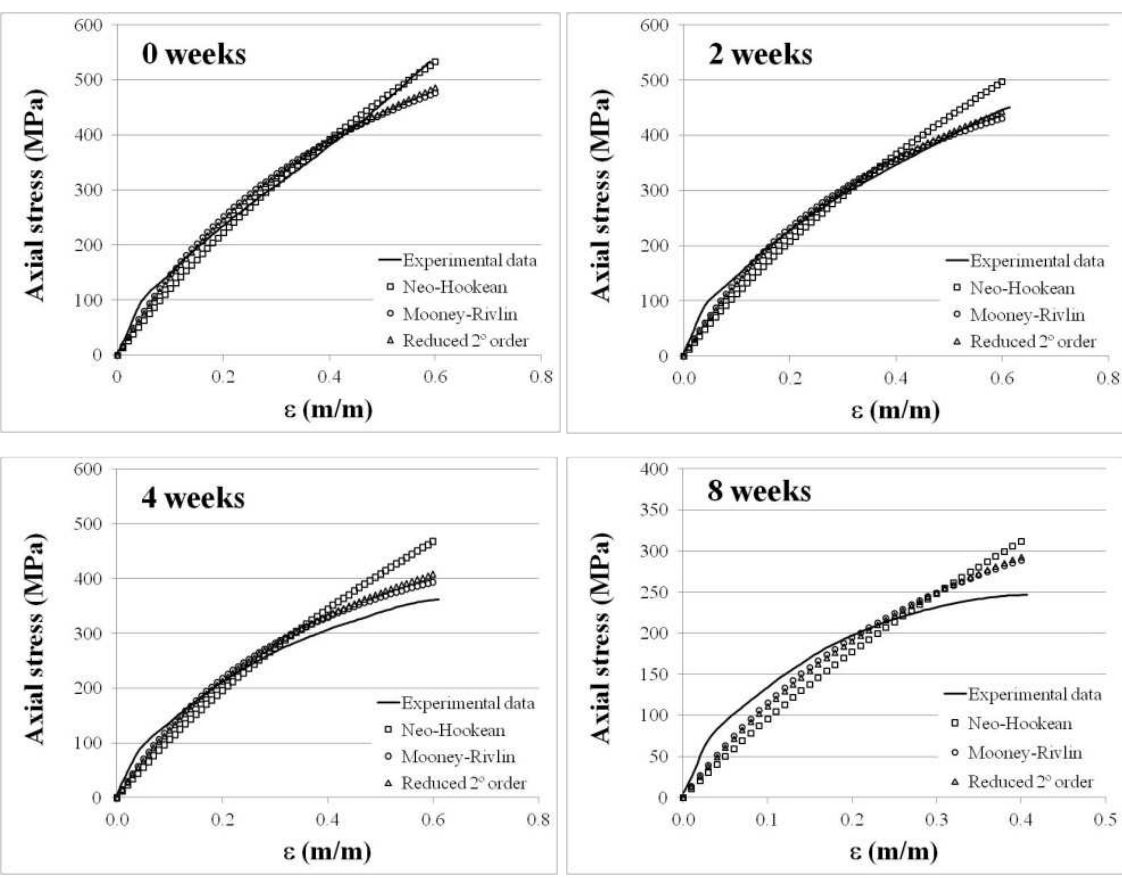

Fig. 12 - Axial nominal stress vs. strain for $0,2,4$ and 8 weeks of degradation (experimental data and material models) (Vieira et al. 2011b)

\section{Implementation and application of the new approach for 4D numerical analysis of scaffolds}

In this section, an example of the new approach for predicting the life-cycle of a hydrolytic degradable device, and its implementation in ABAQUS standard is shown, using the Neo-Hookean material model. This is used to simulate PLA-PCL behavior for fiber geometry. As commented earlier, this implementation was carried out using a subroutine UMAT and the PYTHON language. Although NeoHookean model was less accurate than the other models, it is not so complicate to implement, since it uses only one material parameter $\mu_{l}$. Furthermore, it avoids the violation of the $2^{\text {nd }}$ Law of Thermodynamics, which happens for the other models with negative values for the material parameters $\left(\mu_{2}\right.$ and $\left.\mu_{3}\right)$. For this 3D case, the first and second invariants of deviator part of the left Cauchy-Green deformation tensor are given by:

$\mathrm{I}_{\mathrm{B}}=\operatorname{tr}(\mathrm{B})$

$\mathrm{II}_{\mathrm{B}}=1 / 2\left[(\operatorname{trB})^{2}-\operatorname{trB}^{2}\right]^{1 / 2}$ 
where $B$ is the deviator stretch tensor $\left(B=F F^{T}\right)$. The Neo-Hookean compressible hyper elastic model is given by stored energy function of the form:

$\mathrm{W}=\left(\mu_{1} / 2\right)\left(\mathrm{I}_{\mathrm{B}}-3\right)+\mathrm{G}(\mathrm{J}-1)^{2}$

where $G$ is a material constant that depends on the compressibility ( $G=0$ for incompressible materials). $J$ is the determinant of the deformation gradient ( $J=1$ for incompressible materials):

$\mathrm{J}=\operatorname{det}(\partial \mathrm{x} / \partial \mathrm{X})$

where $x$ is the current 3D position of a material point and $X$ is the reference position of the same point. Then:

$\mathrm{F}=\mathrm{J}^{-1 / 3}(\partial \mathrm{x} / \partial \mathrm{X})$

is the deformation gradient with volume change eliminated. The Cauchy stress tensor for the Neo-Hookean model used in this example is given by:

$$
\mathrm{T}=\left(\mu_{1} / \mathrm{J}\right) \operatorname{dev}(\mathrm{B})+2 \mathrm{C}(\mathrm{J}-1) \mathrm{I}
$$

where $\mathrm{I}$ is the $2^{\text {nd }}$ order identity tensor.

The first material parameter is calculated as function of the hydrolytic damage, $\mu_{l}\left(d_{h}\right)$, according to a linear regression shown in Error! Reference source not found.

In this example, a 3D model of a fiber was developed by means of a script in PYTHON language, using solid and axisymmetric elements, with parabolic interpolation functions, as well as with reduced and/or hybrid integration. This script is run by $\mathrm{ABAQUS}$ and the degradation time is required as an input parameter data (Error! Reference source not found. 13). The hydrolysis rate of the material $(u)$ and the strength of the non-degraded material $\left(\sigma_{0}\right)$ are initially set in the command lines. The material was considered nearly incompressible $\left(G=10^{-3}\right)$. Then the script calculates the hydrolytic damage $\left(d_{h}\right)$ according to Equation 9, and the material strength $\left(\sigma_{t}\right)$ according to Equation 8 , for a given the degradation time $(t)$. The script also calculates the material parameter $\left(C_{10}=\mu_{1} / 2\right)$ as a function of the hydrolytic damage $C_{10}\left(d_{h}\right)$. The material strength $\left(\sigma_{t}\right)$ and the material parameters $\left(C_{10}\right.$ and $G$ ) are considered input data for the UMAT subroutine as shown in Error! Reference source not found.. 


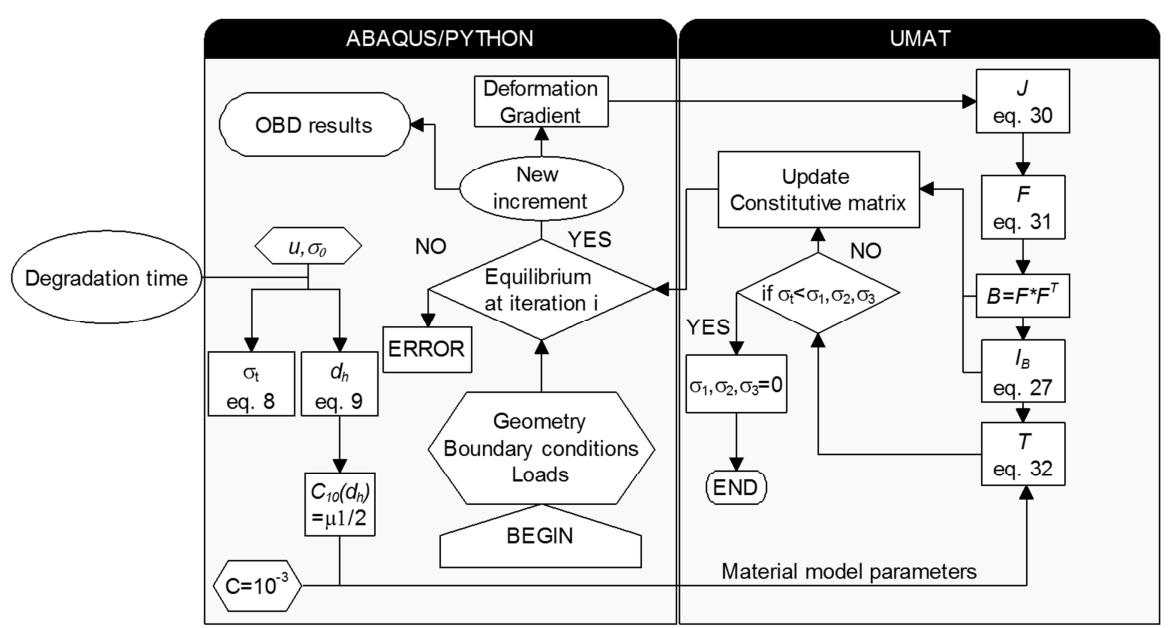

Fig. 13 - Flow of operations done by ABAQUS/PYTHON and the UMAT subroutine (Vieira et al. 2011a)

Based on the geometry, the loadings and boundary conditions, ABAQUS calculates the variables that correspond to the deformation gradient $(\partial \mathrm{x} / \partial \mathrm{X})$. Then, the UMAT calculates the Jacobian ( $\mathrm{J}$ ) and the distortion tensor (F), according to Equation 30 and 31 respectively, for each integration point of the FEM model. The deviator stretch tensor B is then calculated before the calculation of stress Cauchy tensor T, according to Equation 32. The implemented UMAT compares the principal stresses $\left(\sigma_{1}, \sigma_{2}\right.$ and $\left.\sigma_{3}\right)$ to the strength $\left(\sigma_{t}\right)$ for each integration point, acting as a failure criterion. Whenever these are greater than the strength, for a certain increment, the subroutine sets them to zero in the finite element analyzed. Finally, the UMAT constructs the constitutive matrix and calculates the result for each increment into the OBD (Output Base Data) file of ABAQUS. The flow chart of calculi operations is represented in Error! Reference source not found..

Error! Reference source not found. a) shows the mesh of the finite element model and boundary conditions applied, as well as a numerical result for maximum principal stress. The CAX8H (8-node biquadratic axisymmetric quadrilateral, hybrid, linear pressure element) and C3D20RH (20-node quadratic brick, hybrid, linear pressure, reduced integration) element types were used, with similar results. Although the first element type is simpler and faster to calculate, it cannot be used in 3D complex shapes. From Error! Reference source not found. b), one can see that the hyper elastic material model allowed a reasonable approximation of the tensile test results reported previously. For this particular geometry and load conditions, no mesh size dependence was found. Finally, more details can be seen at Vieira et al. (Vieira et al. 2011a). 


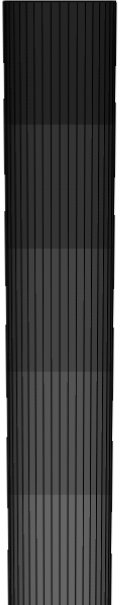

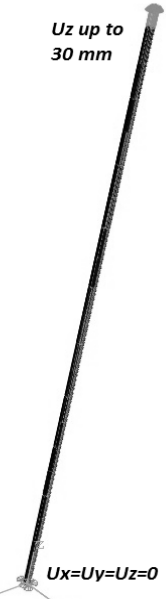

a)

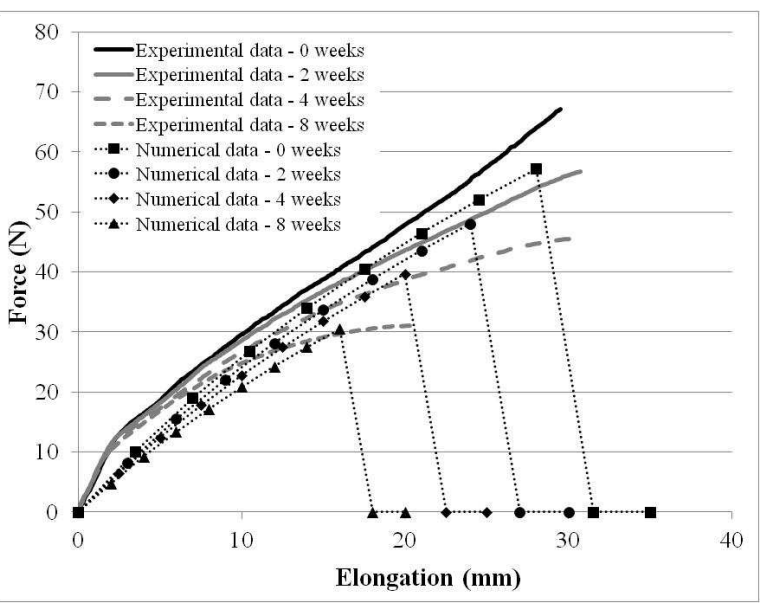

b)

Fig. 15 - (a) 3D model of the fiber; (b) Experimental vs. numerical results according of tensile tests to PLA-PCL fibers at different stages of hydrolytic degradation (Vieira et al. 2011a)

\section{Conclusion}

The numerical approach presented here can be used as design tool of biodegradable polymeric devices with further complex 3D geometries, considering the initial condition of instantaneous diffusion (homogenous degradation along the volume). Although this approach was only tested with this particular blend, the authors believe that this can be extended to other thermoplastic biodegradable materials with response similar to hyper elastic behavior. For example, presently 3D scaffolds can be printed with biodegradable polymers. Using this new approach, complex geometries can be modeled in a commercial 3D drawing software, or digitalized from biomedical images, and then exported to ABAQUS ${ }^{\mathrm{TM}}$ to predict its mechanical behavior during degradation by numerical simulation. This approach, only valid for small thickness devices in the first steps of erosion, without considering the degradation rate dependence on temperature, environment and stress state, can be further expanded to more detailed models that consider these dependencies, the crystalline degree dependence, and the diffusion of water, enzymes and degradation products. In these further complex problems, damage will depend not only on the degradation time, but also on the water concentration and the hydrolysis kinetic constant, no longer constants but time, geometrical, degradation media, temperature and stress state dependents.

The development of better models for biodegradable polymers can enhance the scaffolds design process. The numerical approach presented here, based on the calculation 
of one material parameter of Neo-Hookean hyper elastic model, that is a function of the degradation time, can overcome this limitation and enable a reasonably prediction of the life time of newer and more complex scaffolds.

\section{Acknowledgments}

Volnei Tita would like to thank Research Foundation of State of Sao Paulo (process number: 09/00544-5).

\section{References}

Aslan S, Calandrelli L, Laurienzo P, Malinconico M, Migliares C (2000) Poly(d,l-lactic acid)/poly(caprolactone) blend membranes: preparation and morphological characterization. $\mathrm{J}$ Mater Sci 35:1615-1622

Auras R, Harte B, Selke S (2004) An Overview of Polylactides as Packaging Materials. Macromol Biosci 4:835-864

Bastin G, Dochain D (1990) On-line estimation and adaptative control of bioreactor. Elsevier, Amsterdam.

Bellenger V, Ganem M, Mortaigne B, Verdu J (1995) Lifetime prediction in the hydrolytic ageing of polyesters. Polym Degrad Stab 49:91-97

Bikiaris D, Papageorgiou G, Achilias D, Pavlidou E, Stergiou A (2007) Miscibility and enzymatic degradation studies of poly(e-caprolactone)/ poly(propylene succinate) blends. Eur Polym J 43:2491-2503

Chen G-Q, Wu Q (2005) Review: The application of polyhydroxyalkanoates as tissue engineering materials. Biomaterials 26:6565-6578

Chu CC (1985) Strain-accelerated hydrolytic degradation of synthetic absorbable sutures. In: Hall CW (ed) Surgical Research, Recent Developments: Proceedings of the First Annual Scientific Session of the Academy of Surgical Research. Pergamon Press, San Antonio

Colombo A, Karvouni E (2000) Biodegradable stents: fulfilling the mission and stepping away. Circulation 102:371-373

Endo M, Aida T, Inoue A (1987) Immortal polymerization of e-caprolactone initiated by aluminum porphyrin in the presence of alcohol. Macromolecules 20:2982-2988

Fan LT, Lee Y (1983) Kinetic studies of enzymatic hydrolysis of insoluble cellulose: deriva-tion of a mechanistic kinetic model. Biotechnol Bioeng 25:2707-2733

Fan LT, Lee Y, Beardmore DH (1980) Major chemical and physical features of cellulosic materials as substrates for enzymatic hydrolysis. Adv Biochem Eng 14:101-117

Farrar DF, Gilson RK (2002) Hydrolytic degradation of polyglyconate B: the relationship between degradation time, strength and molecular weight. Biomaterials 23:3905-3912

Garlotta DA (2001) Literature Review of Poly(Latic Acid). J Polym Environ 9:63-84

Göpferich A (1996) Mechanisms of polymer degradation and erosion. Biomaterials 17:103-114

Göpferich A, Langer R (1993) Modeling of polymer erosion. Macromolecules 26:4105-4112

Grizzi I, Garreau H, Li S, Vert M (1995) Hydrolytic degradation of devices based on poly[DLlactic acid) size-dependence. Biomaterials 16:305-311

Han X, Pan J (2009) A model for simultaneous crystallization and biodegradation of biodegradable polymers. Biomaterials 30:423-430

Herzog K, Müller R-J, Deckwer W-D (2006) Mechanism and kinetics of the enzymatic hydrolysis of polyester nanoparticles by lipases. Polym Degrad Stab 91:2486-2498 
Hill CG (1977) An Introduction to Chemical Engineering Kinetics and Reactor Design. John Wiley and Sons, New York

Kennedy JF, Melo EHM (1990) Bioconversions of cellulose - A major source of material for the biochemical industry. Br Polym J 23:193-198

Kirby AJ (1972) Hydrolysis and formation of esters of organic acids. In: Bamford CH, Tipper CFH (eds) Comprehensive Chemical Kinetics, Ester Formation and Hydrolysis and Related Reactions. Elsevier, Amsterdam

Klyosov AA, Rabinowitch M (1980) Enzymatic conversion of cellulose to glucose: present state of the art and potential. Plenum Press, New York

Krynauw H, Bruchmüller L, Bezuidenhout D, Zilla P, Franz T (2011) Constitutive modelling of degradation induced mechanical changes in a polyester-urethane scaffold for soft tissue regeneration. In: Fernandes PR et al. (eds) Proceedings of II International Conference on Tissue Engineering. Lisbon

Levenberg S, Langer R (2004) Advances in tissue engineering. In: Schatten GP (ed) Current topics in developemental biology. Elsevier Academic, San Diego

Li SM, Garreau H, Vert M (1990) Structure-property relationships in the case of the degrada-tion of massive aliphatic poly(a-hydroxyacids) in aqueous media. Part 3: influence of the morphology of poly(L-lactic acid). J Mater Sci, Mater Med 1:198-206

Lunt J (1998) Large-scale Production, Properties and applications of Polylatic Acid Polymers. Polym degrad stab 59:145-152

Lyu SP, Sparer R, Untereker D (2005) Analytical solutions to mathematical models of the surface and bulk erosion of solid polymers. J Polym Sci Part B 43:383-397

Meek M, Jansen K, Steendam R, van Oeveren W, van Wachem P, van Luyn M (2004) In vitro degradation and biocompatibility of poly(DL-lactide-ם-caprolactone) nerve guides. J Biomed Mater Res A 68:43-51

Metzmacher I, Radu F, Bause M, Knabner P, Friess W (2007) A model describing the effect of enzymatic degradation on drug release from collagen minirods. Eur J Pharm Biopharm 67:349-360

Miller ND, Williams DF (1984) The in vivo and in vitro degradation of poly(glycolic acid) suture material as a function of applied strain. Biomaterials 5:365-368

Morrison RT, Boyd RN (1992) Organic chemistry. Prentice Hall, New Jersey

Nair LS, Laurencin CT (2007) Biodegradable polymers as biomaterials. Prog Polym Sci, 32:762-798

Nikolic MS, Poleti D, Djonlagic J (2003) Synthesis and characterization of biodegradable poly(butylene succinate-co-butylene fumarate)s. Eur Polym J 39:2183-2192

Pitt GG, Gratzl MM, Kimmel GL, Surles J, Sohindler A (1982) Aliphatic polyesters II. The degradation of poly(d,l-lactide), poly(e-caprolactone), and their copolymers in vivo. Biomaterials 2:215-220

Saha SK, Tsuji H (2009) Enhanced crystallization of poly(L lactide-co-- caprolactone) in the presence of water. J Appl Polym Sci 112:715-720

Seretoudi G, Bikiaris D, Panayiotou C (2002) Synthesis, characterization and biodegradability of poly(ethylene succinate)/poly(e-caprolactone) block copolymers. Polymer 43:5405-5415

Shen-Guo W, Bo Q (1992) Polycaprolactone-poly(ethylene glycol) block copolymer, I: synthesis and degradability in vitro. Polym Adv Tech 4:363-368

Siparsky GL, Voorhees KJ, Miao F (1998) Hydrolysis of polylactic acid (PLA) and polycaprolactone (PCL) in aqueous acetonitrile solutions: autocatalysis. J Environ Pol Degrad 6:31-41

Soares JS, Rajagopal KR, Moore JE (2010) Deformation induced hydrolysis of a degradable polymeric cylindrical annulus. Biomech Model Mechan 9:177-186

Södergard A, Stolt M (2002) Properties of lactic acid based polymers and their correlation with composition. Prog Polym Sci 27:1123-1163

Sykes P (1975) A Guidebook to Mechanism in Organic Chemistry. Longman Group Ltd, London

Tamela TL, Talja M (2003) Biodegradable urethral stents. BJU Int 92:843-850 
Therin M, Christel P, Li SM, Garreau H, Vert M (1992) In vivo degradation of massive poly(ahydroxyacids): validation of in vitro findings. Biomaterials 13:594-600

Tokiwa Y, Suzuki T (1977) Hydrolysis of polyesters by lipase. Nature 270:76-78

Tsuji H, Ikada Y (1998) Properties and morphology of poly(Llactide).II. Hydrolysis in alka-line solution. J Polym Sci Part A 36:59-66

Tsuji H, Ikada Y (2000) Properties and morphology of poly(Llactide) 4. Effects of structural parameters on long-term hydrolysis of poly(L-lactide) in phosphate-buffered solution. Polym Degrad Stab 67:179-189

Tsuji H, Nakahara K (2001) Poly(L-lactide)—IX hydrolysis in acid media. J Appl Polym Sci 86:186-194

Tzafriri AR, Bercovier M, Parnas H (2002) Reaction Diffusion Model of Enzymatic Erosion of Insoluble Fibrillar Matrices. Biophys J 83:776-793

Van Krevelen DW (1976) Properties of Polymers. Elsevier, Amsterdam

Vert M, Li S, Garreau H (1991) More about the degradation of LA/GA-derived matrices in aqueous media. J Control Rel 16:15-26

Vieira AC, Guedes RM, Marques AT (2009) Development of ligament tissue biodegradable devices: A review. J Biomech 42:2421-2430

Vieira AC, Marques AT, Guedes RM, Tita V (2011a) Material model proposal for biodegradable materials. Procedia Engineering 10:1597-1602

Vieira AC, Vieira JC, Ferra JM, Magalhães FD, Guedes RM, Marques AT (2011b) Mechanical study of PLA-PCL fibres during in vitro degradation. J Mech Behav Biomed 4:451-460

von Burkersroda F, Schedl L, Göpferich A (2002) Why biodegradable polymers undergo surface or buk erosion. Biomaterials 23:4221-4231

Walker LP, Wilson DB (1991) Enzymatic hydrolysis of cellulose: an overview. Bioresource Tech 36:3-14

Wang Y, Pan J, Han X, Sinka C, Ding L (2008) A phenomenological model for the degradation of biodegradable polymers. Biomaterials 29:3393-3401

Ward I (1983) Mechanical Properties of Solid Polymers. Wiley \& Sons, Chichester

Yu R, Chen H, Chen T, Zhou X (2008) Modeling and simulation of drug release from multilayer biodegradable polymer microstructure in three dimensions. Sim Model Pract Th 16:1525

Zhang X, Espiritu M, Bilyk A, Kurniawan L (2008) Morphological behaviour of poly(lactic acid) during hydrolytic degradation. Polym Degrad Stab 93:1964-1970 